\title{
Journey 'Round the Sun: STEREO Science and Spacecraft Performance Results
}

\author{
Daniel A. Ossing \\ The Johns Hopkins University \\ Applied Physics Laboratory \\ 11100 Johns Hopkins Road \\ Laurel, MD 20723 \\ 240-228-8319 \\ Dan.Ossing@jhuapl.edu
}

\author{
Therese A. Kucera \\ Georgia A. de Nolfo \\ David A. Quinn \\ NASA GSFC \\ 8800 Greenbelt Rd \\ Greenbelt, MD 20771 \\ 301- 286-6681 \\ Therese.A.Kucera@nasa.gov
}

\begin{abstract}
The Solar TErrestrial RElations Observatory (STEREO) was originally designed as a two to five year heliocentric orbit mission to study coronal mass ejections (CMEs), solar energetic particles (SEPs), and the solar wind. After over ten years of continuous science data collection, the twin NASA STEREO observatories have significantly advanced the understanding of Heliophysics. This mission was the first to image CMEs all the way from the Sun to Earth and to observe the entire sphere of the Sun at one time. STEREO has demonstrated the importance of a point of view beyond the Sun-Earth line to significantly improve CME arrival time estimates and in understanding CME structure and trajectories and the longitudinal distribution of SEPs. STEREO was also the first to use one launch vehicle to insert two spacecraft into opposing heliocentric orbits, undergo a 3.5 month long superior solar conjunction, implement unattended daily science operations on two deep space observatories, maintain 7 arcsec continuous pointing without gyros, and detect and attempt to recover a spacecraft after a 22-month long communications anomaly at a range of $2 \mathrm{AU}$. This paper discusses the significant performance results after the first ten years of operations of the STEREO mission from its journey around the Sun.
\end{abstract}

\section{TABLE OF CONTENTS}

1. INTRODUCTION 1

2. MISSION OVERVIEW 1

3. INSERTING OBSERVATORIES IN OPPOSING HELIOCENTRIC ORBITS USING ONE LAUNCH VEHICLE .2

4. UNATTENDED OPERATIONS OF TWO DEEP SPACE OBSERVATORIES 2

5. Continuous Fine Pointing Without Gyros 3

6. SURVIVING A 3.5 MONTH LONG SOlAR CONJUNCTION

7. LOSS OF AND EFFORTS TO RECOVER THE STEREO BEHIND OBSERVATORY .

8. LESSONS LEARNED ..................................................... 9

9. STEREO SCIENCE ............................................ 10

10.CONCLUSION .......................................................... 13

11.ACKNOWLEDGEMENTS..................................... 13

12.REFERENCES ................................................... 13

\section{INTRODUCTION}

The STEREO mission consists of two nearly identical spacecraft orbiting the Sun, combining both in situ and remote sensing instrumentation to expand our understanding the sun and solar wind beyond what can be gleaned with a single vantage point. This mission has produced unique scientific contributions and also unique challenges. Here we describe some of the significant engineering challenges confronted during mission operations and also some of the scientific highlights of the mission. As those that do not learn from history are doomed to repeat the mistakes, lessons learned are presented.

\section{MISSION OVERVIEW}

STEREO (Solar-TErestrial RElations Observatory) [1] is the third mission in the Solar Terrestrial Probes program of the National Aeronautics and Space Administration (NASA) Science Mission Directorate Sun-Earth Connection theme. The mission consists of two nearly identical spacecraft in heliocentric orbits designed to investigate the threedimensional structure of the Sun's corona, the origin of coronal mass ejections (CME's), and the dynamic coupling between CME's and the Earth's environment. This Heliophysics mission is managed by NASA Goddard Space Flight Center (GSFC).

The two spacecraft were designed, integrated, and operated by Johns Hopkins University/Applied Physics Laboratory (JHU/APL) with instrument packages provided by the Naval Research Laboratory, University of California Berkeley, University of New Hampshire, the Observatoire de Paris and their partners. The instrumentation includes a mix of remotes sensing in visible, extreme ultraviolet and radio wavelengths and in-situ detection of conditions at the spacecraft.

- Sun-Earth Connection Coronal and Heliospheric Investigation (SECCHI) - imaging suite including five telescopes that, combined, image from the Sun to Earth orbit. 
- In situ Measurements of Particles and CME Transients (IMPACT) - instrument suite including in situ measurements of the solar wind, including magnetic field, and energetic particles.

- Plasma and Suprathermal Ion Composition (PLASTIC) - characteristics of the in situ solar wind, including low energy ions.

- $\quad$ STEREO WAVES (SWAVES) - radio waves and in situ electric fields.

\section{INSERTING OBSERVATORIES IN OPPOSING HELIOCENTRIC ORBITS USING ONE LAUNCH VEHICLE}

The twin STEREO observatories were successfully launched stacked, with the Behind observatory on the bottom, on a single Boeing Delta II 7925-10L rocket on 26 October 2006 Coordinated Universal Time (UTC) from the Kennedy Space Center in Florida. The complex first orbit operations during the 10 days following launch, including two $0.2 \mathrm{~m} / \mathrm{s}$ engineering test maneuvers and an $11.7 \mathrm{~m} / \mathrm{s}$ apogee maneuver to raise perigee, preventing atmospheric reentry, were described in a previous paper [2]. The highly accurate launch inserted the two spacecraft into highly elliptical Earth orbits with apogee beyond lunar orbit. Phasing orbits were employed allowing periodic maneuvers needed over a two-month period to target the lunar swingbys to accomplish the mission objectives thereby placing the observatories into heliocentric orbits; with STEREO Ahead's orbit slightly inside Earth's (and therefore slightly faster than Earth), and with STEREO Behind's orbit slightly outside Earth's (and therefore slightly slower than Earth), see Figure 1.

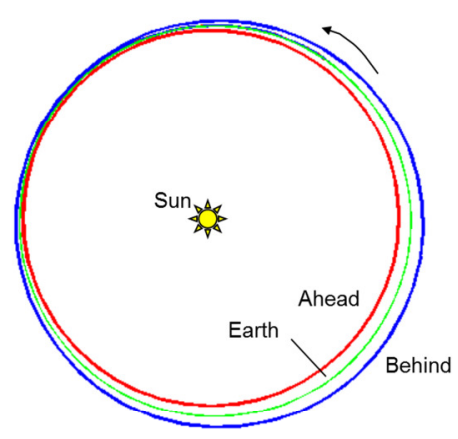

Heliocentric Inertial Coordinates (Ecliptic Plane Projection) calibrate several instruments before the prime science mission began. STEREO was the first mission to use lunar swingbys to place two spacecraft, launched on one launch vehicle, into very different (in this case, oppositely-directed) orbits. Maneuvers that were performed extremely accurately, combined with an accurate launch, has left the STEREO spacecraft with a generous supply of propellant sufficient to manage momentum for over a 100 years.

The resulting orbits cause the two satellites to move away from the Earth at a rate of $22^{\circ}$ per year, as required by the mission. See Figure 2 below. Noting the direction of the Earth's revolution about the Sun, the positions of STEREO Ahead and STEREO Behind relative to the Earth gives rise to the nicknames "Ahead" and "Behind," respectively, for the two spacecraft.

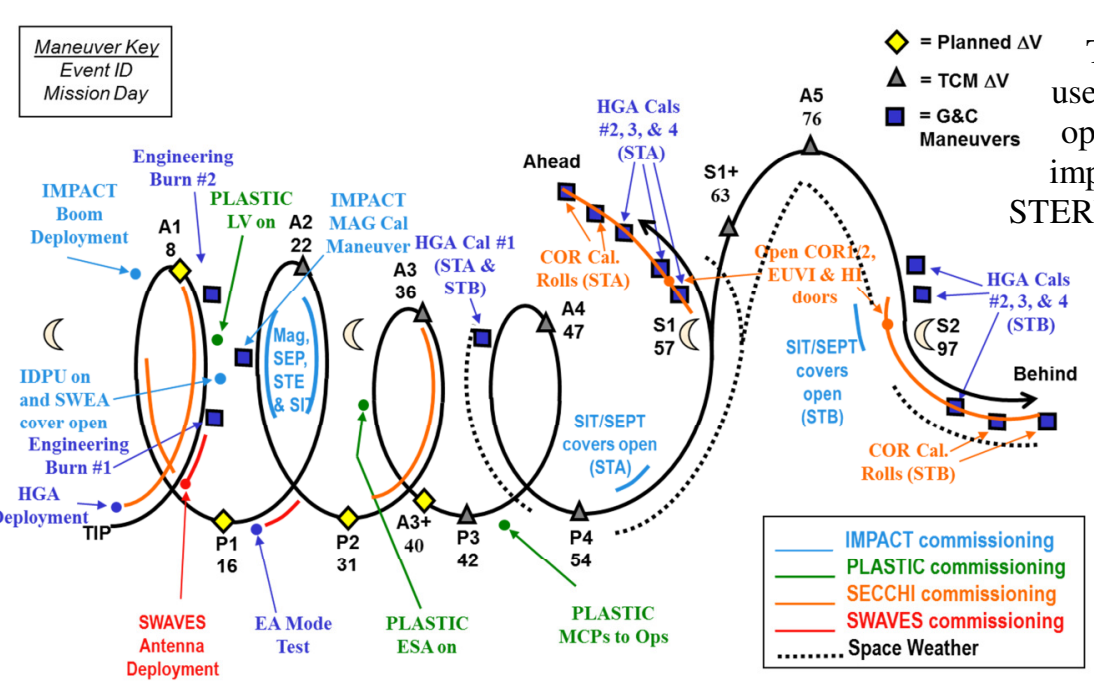

Figure 1: Schematic of STEREO's Phasing Orbits showing key events

The two-month phasing orbits also provided the opportunity to check out the spacecraft subsystems and instruments and

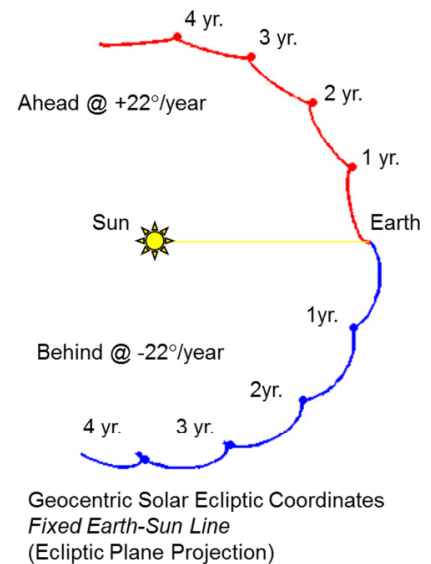

Figure 2: STEREO Orbits

\section{UNATTENDED OPERATIONS OF TWO DEEP SPACE OBSERVATORIES}

The STEREO mission operations center (MOC) uses track automation for unattended control operations. This operations concept was implemented six months after launch. The STEREO mission operates as two continuously recording solar data pipelines with daily Deep Space Network (DSN) and/or European Space Agency (ESA) tracks for science data return. The mission also employs a decoupled instrument operations concept where each instrument team operates their respective instruments and routinely sends commands on a daily basis. Each observatory uses rule based logic for on board fault protection as the first line of defense. For these reasons, an unattended control operations concept was developed which resulted in minimizing the phase E staffing level as there is no control team. 
The DSN and ESA deep space antennas are used for tracking, and operate behind a firewall on a secure network. There is a separate, but less secure network that allows for remote notification, which is also used for planning and assessment but cannot send commands. Control information is relayed from the secure to the less secure network in realtime. The two networks meant that the STEREO track automation system would need to be split into two sections. First would be the Master Control Procedure (MCP) to perform the mechanics of configuring to the correct DSN or ESA station and sending commands would be on the secure network. A separate paging system known as Tron performs state of health checks on the spacecraft on the less secure network. Telemetry is used to communicate between the two systems through database global variables. These are essentially null values in the telemetry database that can be set by the ground.

Given this baseline hardware and software configuration, a low-tech implementation using Satellite Test Operations Language (STOL) was used for track automation. STOL is an interpreted language similar to Basic and was familiar to members of the mission operations team. This allowed for ease of use and maintainability by the missions operations team (MOT) over the course of the mission as events warrant. Specially developed software applications generally require software staff to maintain them, using STOL reduced software maintenance. The primary driver of the track automation software is the DSN 7-day Schedule, which also contains ESA tracks. The mission planning software creates a command procedure that sets database global variables on contact information for the next 21 days.

The MCP supports limited failure recovery actions for which the MOT is notified via text and or email messages. Most of the MCP failure recovery actions deal with problems establishing a command link.

While the MCP executes in a linear fashion, the Tron program does not. Tron was therefore implemented as a scanning program. Every 30 seconds it performs a complete scan of the MCP status, network status, as well as spacecraft status. If it identified that a track was in progress it would perform real-time checks. Tron implements a core set of checks that are performed continuously during a track, and a set of extended checks that are performed at the start of the track and hourly thereafter. Core checks are limited to the following:

- Fault autonomy rule firing

- Spacecraft not in the operational mode

- Battery state of charge less than $95 \%$

- High Gain Antenna (HGA) pointing is disabled

- No command acknowledgment for any command

- No telemetry received for any 20 minute period

- Unresponsive networks

- Unresponsive MCP
The extended checks grew from the health \& safety checking procedure used at launch and checks over 2000 telemetry points. A failure in any check could be responded to by either an email or a text message.

Responsibility for monitoring the automation system and responding to pages rotates through the team on a weekly basis. Once paged, team members can remotely log in from their remote location and monitor spacecraft telemetry. Once the problem is determined, the rest of the team is advised with the status/resolution of the problem via email. Typical issues are failures with the command or telemetry links at the station. The team may get two pages every week.

\section{Continuous Fine Pointing Without GYROS}

The longevity design requirement for STEREO was just two years, with a design goal of five years. Thus, both STEREO spacecraft have greatly exceeded expectations. Most hardware has shown no aging effects at all. However, the gyroscopes in the Inertial Measurement Units (IMU) and the ST have some issues. Each spacecraft is equipped with a pair of IMUs, with each IMU containing three orthogonal ring laser gyroscopes (RLGs) and three orthogonal accelerometers. The original operational concept was to fly with one IMU on at all times to provide $100 \mathrm{~Hz}$ rate data for maintaining continuous pointing requirements of $\leq 7.25$ arcsec, listed in Table 1.

Table 1. STEREO Pointing Performance Requirements

\begin{tabular}{|c||c|c|c|}
\hline & $\begin{array}{c}\text { Pitch and } \\
\text { Yaw } \\
\text { Performance } \\
\text { Each Axis }\end{array}$ & $\begin{array}{c}\text { Roll } \\
\text { Performance } \\
\text { (arcmin) }\end{array}$ & $\begin{array}{c}\text { Window } \\
\text { Times } \\
\text { (seconds) }\end{array}$ \\
\hline \hline Accuracy & $3 \sigma \mathrm{a} \leq 7.25$ & $3 \sigma \mathrm{a} \leq 35$ & ---- \\
\hline \multirow{2}{*}{ Jitter } & $\sigma \mathrm{j} \leq 1.53$ & $\sigma \mathrm{j} \leq 3.4$ & $\begin{array}{c}\mathrm{Tj}=15(30 \\
\text { as a goal) }\end{array}$ \\
\hline \multirow{2}{*}{$\begin{array}{c}\text { Windowed } \\
\text { Stability }\end{array}$} & $\sigma \mathrm{sw} \leq 1.90$ & $\sigma \mathrm{sw} \leq 6.70$ & $\begin{array}{c}\mathrm{T}_{\mathrm{S}}=10.2 \\
\mathrm{~T}_{\mathrm{j}}=0.1\end{array}$ \\
\cline { 2 - 4 } & $\sigma \mathrm{sw} \leq 3.75$ & $\sigma \mathrm{sw} \leq 6.70$ & $\begin{array}{c}\mathrm{T}_{\mathrm{S}}=25.2 \\
\mathrm{~T}_{\mathrm{j}}=1.0\end{array}$ \\
\hline
\end{tabular}

The second IMU unit was to be kept powered off as a cold spare. In the event of an IMU fault, rule based fault protection would autonomously swaps to the backup IMU.

As gyros aged (and failed) the mission was forced to change to a new concept of operations called Reduced Gyro Operations (RGO), see Figure 3. In RGO, the spacecraft "flies" on the star tracker (ST), sun sensors, and the Guide Telescope (essentially, a high-precision sun sensor), with both IMUs turned off. As the primary IMU had failed or the remaining lifetime was being conserved, the backup IMU 
was turned on only for monthly thruster-firing for momentum management and periodic instrument calibration rolls. This changed the IMU duty cycle from $100 \%$ to less than $3 \%$, thus prolonging the remaining life of the backup IMUs.

The change required some adjustment of parameter values in the attitude determination and control software, as well as new onboard autonomy policies to maintain spacecraft health. For example, if the ST were to suddenly lose lock on stars, the system would need to autonomously power-up its only remaining fully-functional IMU.
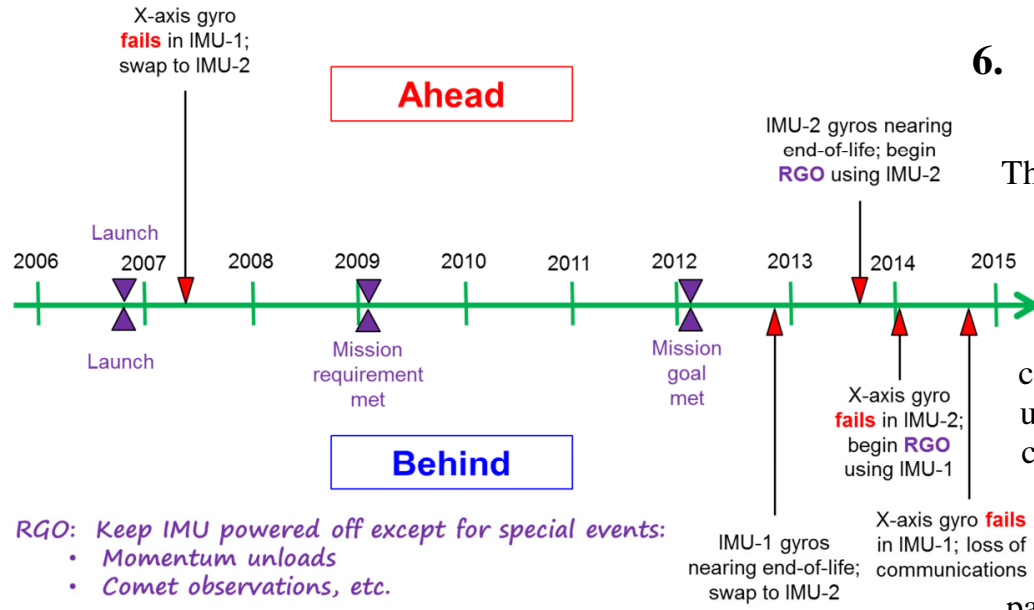

$\begin{array}{cc}\text { X-axis gyro fails } \\ \text { IMU-1 gyros } & \text { in IMU-1; loss of }\end{array}$ nearing end-of-life; swap to IMU-2

\section{Figure 3. Actual IMU history forcing Reduced Gyro Operations concept}

STEREO Ahead switched to RGO in September 2013 and STEREO Behind in February of 2014. There was some observed degradation in pointing performance without gyro measurements, but the system was still able to meet or exceed its pointing requirements. From this point forward, both spacecraft were operating with one failed IMU, and one seriously aged IMU, using RGO to preserve the life of the remaining functional IMU.

Each spacecraft is equipped with a single ST which provides rate data at $10 \mathrm{~Hz}$. With the IMUs in a precarious state, the $\mathrm{ST}$ is relied upon much more than during the early years of the mission. By and large, the ST has performed extremely well. There have been a handful of cases, however, where the ST "dropped lock," i.e., failed to provide attitude solutions for extended periods of time (hours). In most cases, no root cause for the outages has been determined. In each case, eventually, the ST resumed normal operations. This phenomenon was dubbed the "lazy ST."

There is a known aging issue with this ST: its chargecoupled device (CCD) can accumulate radiation damage after years in space. The vendor had advised that lowering the CCD temperature can mitigate this problem. The ST is equipped with a Thermal Electric Cooler (TEC) to keep the $\mathrm{ST}$ at or below room temperature. The set point for the TEC was lowered on the Ahead observatory from $10 \mathrm{deg} \mathrm{C}$ to
-10 deg C in Electrically Erasable Programmable Read-Only Memory (EEPROM) in December of 2014.

To preserve the remaining IMU lifetime on the Ahead observatory, no-gyro operations (NGO) were implemented in December of 2015. Under NGO, the IMUs remain off unless needed by fault protection for safing events and for high value science opportunities, e.g., comet observations, as deemed necessary by the project scientist. Monthly momentum management and periodic instrument calibration rolls are all executed without IMU usage.

\section{Surviving a 3.5 Month Long Solar CONJUNCTION}

The solar conjunction mission phase for STEREO was not initially required by NASA. Nevertheless, after 8 years post launch and multiple mission extensions, it became necessary to safely conduct superior solar conjunction and to continue science return post conjunction. A detailed understanding of the STEREO superior solar conjunction operations can be found in previous paper [3].

communications As suggested by Figure 2, the two spacecraft pass behind the Sun (superior solar conjunction). Xband communications are significantly degraded when the Sun-Probe-Earth (SPE) angle approaches $3^{\circ}$ due to solar scintillation effects. It took Ahead about 3.5 months to pass through this blackout region, and about 2 months for Behind.

These unprecedented months-long solar conjunctions presented two significant problems: how to ensure spacecraft safety when it is designed for daily communications and how to prevent damaging the parabolic HGA as it cannot be used for communications within several degrees of Sun.

\section{Command-Loss Timer}

STEREO is equipped with both a software command-loss timer (SCLT) and a hardware command-loss timer (HCLT). Both the timeout value and the corresponding action taken for the SCLT may be changed by mission operators. The SCLT may be disabled, however, the HCLT is implemented in hardware/firmware and it cannot be disabled. Neither the HCLT timeout value nor the action taken can be changed after launch.

The STEREO HCLT expires if no command is received from the ground within three days (72.332 hours, to be precise). This value was chosen based on the requirements for the immediate post-launch mission phase, where orbital maneuvers might be required every few days in order to avoid Earth impact and to achieve eventual heliocentric orbit. The value is also consistent with the STEREO 
mission concept of operations, which calls for daily station contacts.

Among the many actions taken in response to an HCLT timeout, are:

- Shutdown of all four scientific instrument suites

- Avionics reset, including rebooting of the flight processors

- Reboot the transponder in the communications subsystem

- $\quad$ Power off the Star Tracker (ST)

- Adjust heaters for low-power consumption

As can be seen, collectively, these are drastic actions to take, and not without risk to spacecraft health. Therefore, an HCLT timeout is something to be avoided. In fact, in the almost 8 years of operation prior to initiation of solar conjunction planning, no HCLT resets occurred on either spacecraft.

The STEREO HCLT design, while necessary and appropriate for early operations, presents a serious problem for surviving the solar conjunction. The Ahead spacecraft, for example, experienced 35 HCLT timeouts during its long 3.5 month communications blackout.

\section{Antenna Thermal Issue}

STEREO uses a $1.2 \mathrm{~m}$ parabolic dish as its High Gain Antenna (HGA). This is the spacecraft's primary means of communication. By rotating the HGA gimbal, the flight software maintains the HGA boresight on the Earth as the spacecraft proceeds on its path around the Sun.

As the spacecraft approached superior solar conjunction, sunlight fell more directly into the parabolic dish and was concentrated at the focus. Despite the dish's is flat black surface, this effect drove the antenna feed temperature to the point where damage is possible, see Figure 4. Specifically, after analyzing the materials on the antenna feed, it was concluded that degradation would start around $150^{\circ} \mathrm{C}$, and actual damage would occur around $170^{\circ} \mathrm{C}$. Projections indicated that if the HGA was allowed to point at the Earth through the solar conjunction, the temperature would greatly exceed the damage point.

This thermal issue was not analyzed during the design phase because the goal for mission life was set at five years, and the issue did not arise until mission year eight. While it may seem short-sighted to stop the analysis at five years, it must be remembered that development managers are typically hard pressed to justify costs incurred when attempting to significantly exceed agreed upon mission requirements.

The antenna thermal issue presented one of the biggest challenges for operating STEREO through the solar

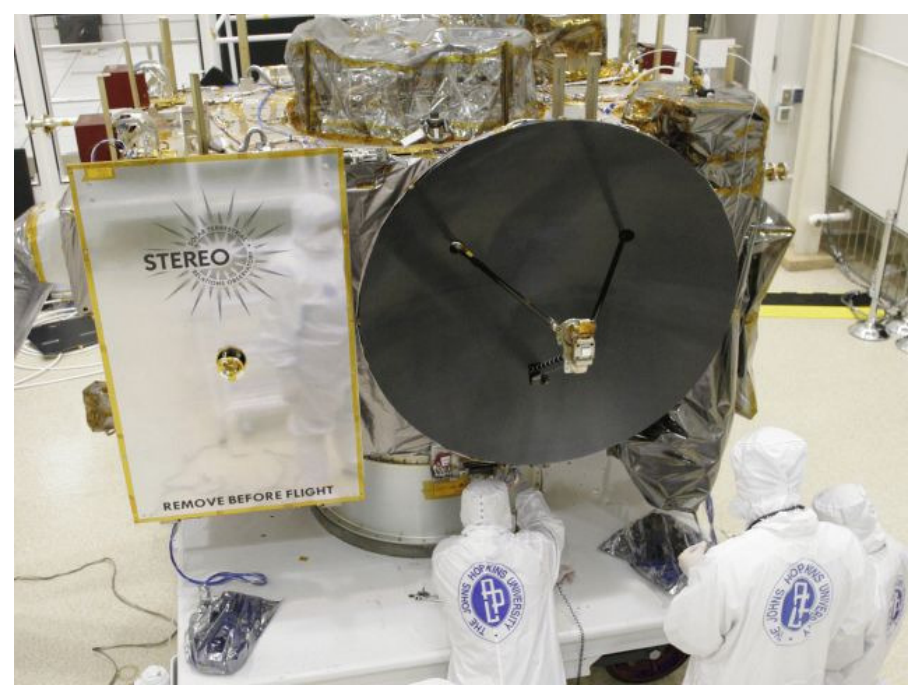

Figure 4. STEREO High Gain Antenna 1.2m parabolic dish

conjunction period. In fact, the issue presented a problem for many months before the actual conjunction and continued for many months after the conjunction. Altogether, the "hot zone" for HGA operations spans up to 15 months (447 days) on Ahead, and 13 months (397 days) on Behind.

\section{Side Lobe Operations and Radio Frequency Communications}

$(R F)$

After data analysis and testing on each spacecraft it was shown that moving the HGA boresight away from the Sun by just a few degrees had a dramatic beneficial effect on the feed temperature. By using the HGA side lobes, a level of radio frequency (RF) communication could be maintained with the spacecraft and reduce thermal effects. Testing revealed that both the command and telemetry links could be maintained by switching from the main lobe of the HGA antenna pattern to either the first or second side lobes, located about $\sim 3^{\circ}$ and $\sim 6^{\circ}$, off the boresight, see Figure 5 . The big drawback to using the side lobes is that the signal strength drops by 1 to 3 orders of magnitude. This forced the implementation of significantly reduced data rates and requires the use of DSN 70 meter ground stations for daily contacts.

\section{Solar Conjunction Plan and Implementation}

The solar conjunction mission phase consisted of three periods of operations; two side lobe operations periods, before and after solar conjunction, and the actual superior solar conjunction period. Side lobe operations are required for several months before and after solar conjunction to maintain the HGA feed assembly temperature to within measureable limits while maintaining some level of groundcommunications. The Table 2 lists the approximate duration of each period. A timeline depicting the significant events the Ahead observatory during the solar conjunction mission phase is shown in Figure 6. 
Off-Boresight Angle (Degrees)

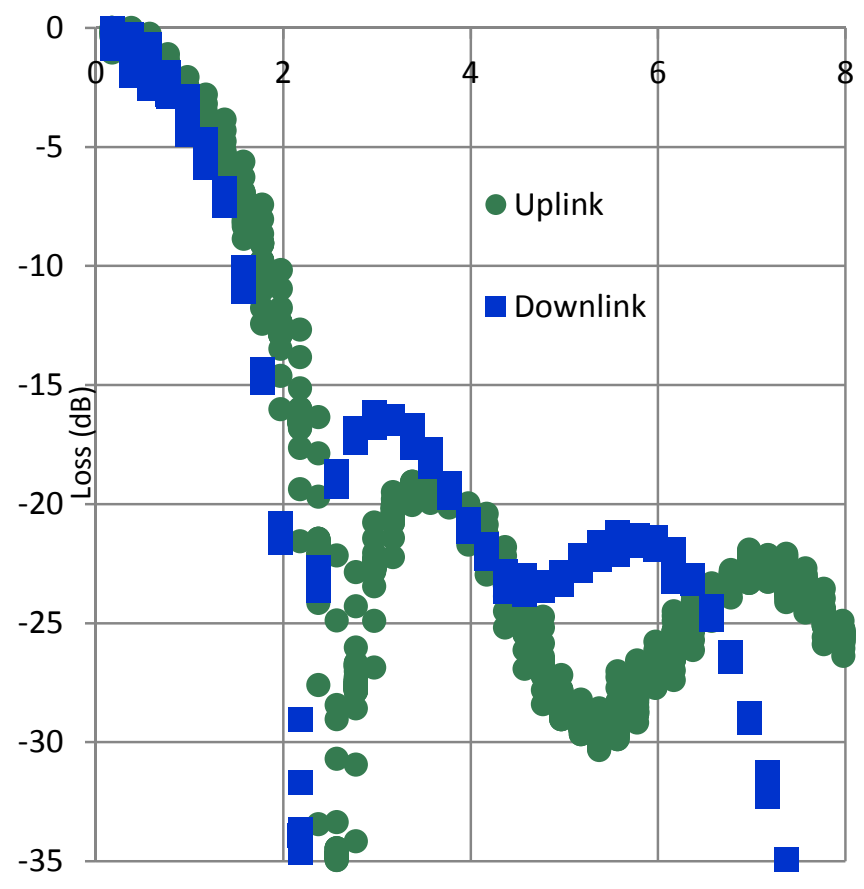

Figure 5. STEREO Ahead High Gain Antenna (HGA) pattern, showing side lobes

The entry and exit into the solar conjunction was at the $2^{\circ}$ SPE angle to ensure communication and verify the required observatory configuration. System momentum was set to 8 Nms to minimize the need for autonomous momentum dumps during the solar conjunction blackout period. Upon entry into the superior solar conjunction, each observatory was commanded, via an absolute time command in the onboard startup sequence, to rotate the spacecraft about the Sun-line at 5 degrees/minute to distribute system momentum so as to better manage buildup along all three axes. As the 72.332 hour HCLT cannot be changed, each observatory undergoes a system reset every three days, thereby reinitializing the state of the observatory. As the SWAVES instrument uses minimal power and its default power-on configuration provides routine science, it remained on and was power-cycled with each three day HCLT system reset to continue to record science data at 1 packet per minute. The other instruments were turned off during the conjunction period.

On June 26, 2015, the first telemetry was received from STEREO Ahead post superior solar conjunction after the $31^{\text {st }}$ HCLT initiated system reset at a $1.5^{\circ}$ SPE angle. The DSN received a strong downlink signal and telemetry using the Goldstone 70 meter station. The observatory was operating nominally continuing to rotate slowly while communicating on the $2^{\text {nd }}$ HGA side lobe as planned. The HGA feed temperature was within the predicted range at $113^{\circ} \mathrm{C}$. System momentum was at the anticipated level of 8.0 Nms. As occurred during solar conjunction entry, after the $31^{\text {st }}$ HCLT reset, the star tracker was again "lazy" to reacquire a solution causing IMU2 to power on for two hours. One additional anomaly occurred, reaction wheel \#3 was not responding to guidance and control (G\&C) torque commands. The observatory continued to rotate nominally as it was designed to do so with only three reaction wheels. Because the observatory was healthy, contingency response to this G\&C anomaly was delayed until July $8^{\text {th }}$ to allow for more reliable communications when the SPE angle reached $2^{\circ}$. Starting on June $29^{\text {th }}$, a three hour DSN 70 meter station tracked the Ahead observatory on a daily schedule as it continued to switch antennas each day and RF performance was found to be highly dependent on solar activity. Due to solar interference, telemetry frame reception varied widely from 13 to $94 \%$ on the HGA. While carrier lock was received on the low gain antennas (LGA), telemetry lock was never successful on the LGAs due to the low SPE angle.

Table 2. Solar Conjunction Mission Phase Durations

\begin{tabular}{|c|c|c|c|}
\hline & $\begin{array}{c}\text { Pre-Solar } \\
\text { Conjunction } \\
\text { (days) }\end{array}$ & $\begin{array}{c}\text { Superior } \\
\text { Solar } \\
\text { Conjunction } \\
\left( \pm 2^{\circ}\right) \text { (days) }\end{array}$ & $\begin{array}{c}\text { Post-Solar } \\
\text { Conjunction } \\
\text { (days) }\end{array}$ \\
\hline $\begin{array}{c}\text { Ahead } \\
\text { Observatory }\end{array}$ & 216 & 105 & 126 \\
\hline $\begin{array}{c}\text { Behind } \\
\text { Observatory }\end{array}$ & 52 & 60 & 285 \\
\hline
\end{tabular}

At 0000 UTC on July $7^{\text {th }}$, the Ahead observatory returned to nominal 3 -axis attitude control as planned and after the $35^{\text {th }}$ and last HCLT initiated system reset on July $8^{\text {th }}$, post superior conjunction recovery operations began. Daily DSN communications on the HGA $2^{\text {nd }}$ side lobe were resumed using an eight hour daily track for six weeks to playback the spacecraft data for analysis. Over the next few days, instruments were powered on and configured for continuing side lobe operations and spacecraft bus recovery operations were completed.

During the post solar conjunction recovery operations on the HGA $2^{\text {nd }}$ side lobe, July $8^{\text {th }}$ through $20^{\text {th }}$, the command success rate varied from $65 \%$ to $100 \%$ depending on solar activity. During this time, the SPE angle increased from 2.0 to $2.5^{\circ}$. While the downlink signal strength was very good during this time, the success rate for telemetry frames received also varied from $76 \%$ to $99 \%$ using the DSN 70 meter stations.

On November $9^{\text {th }}$, the Ahead observatory resumed operations using the main lobe of the HGA resulting in a 13 $\mathrm{dB}$ signal increase. The HGA rode along a $1^{\circ}$ offset from the center of the main lobe till December $30^{\text {th }}$ to keep the HGA feed assembly cool. This increased the HGA feed assembly temperature to $119^{\circ} \mathrm{C}$, which was within the predicted range. 


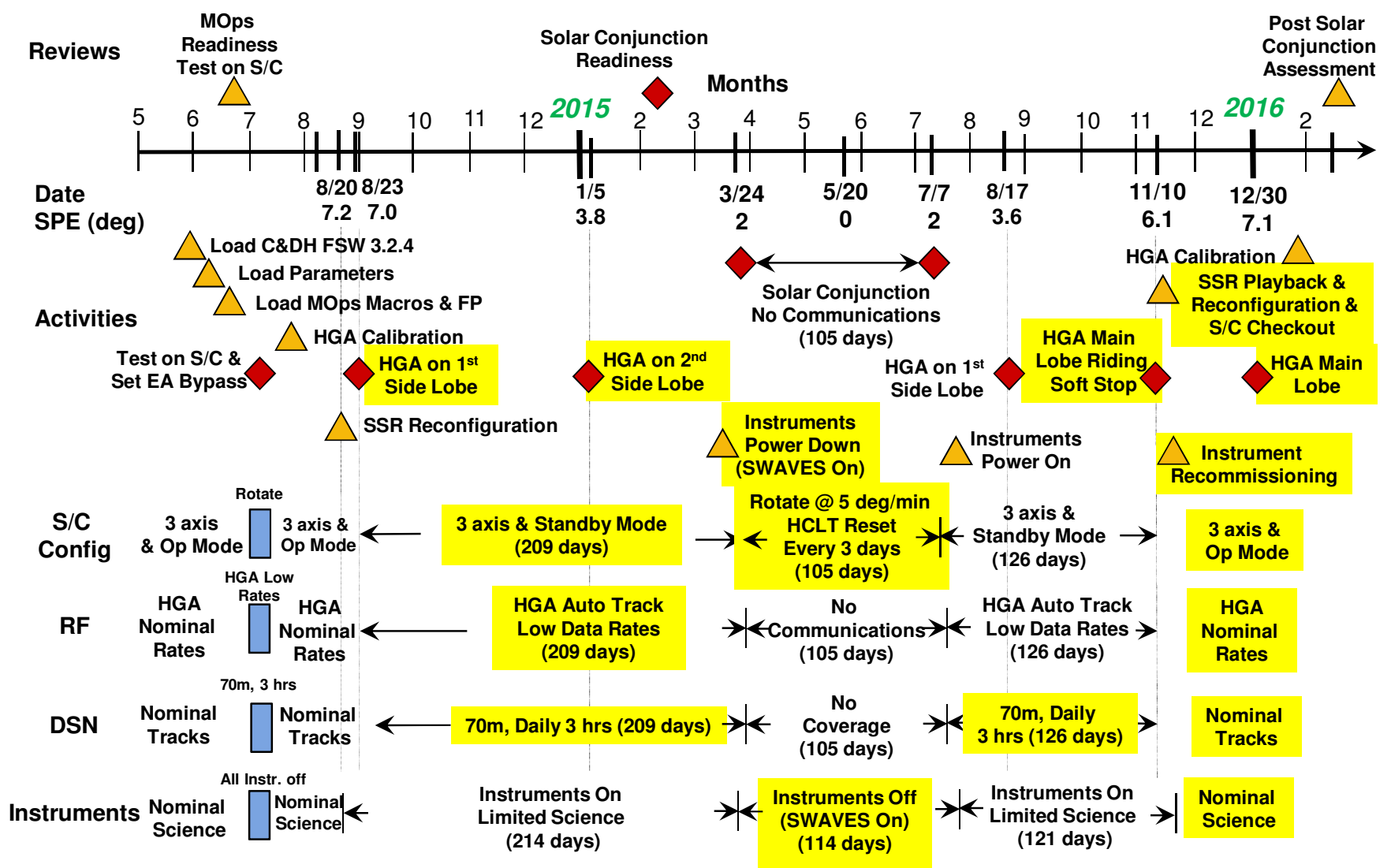

Figure 6. STEREO Ahead Timeline for Superior Solar Conjunction. Highlighted events are those that were tested on-orbit before superior solar conjunction

Even at a $1^{\circ}$ offset, data rates increased significantly, 240 kbps on the downlink and 2000 bps on the uplink. The downlink rate gradually returned to the highest rate, 720 kbps, when communication on the center of the HGA main lobe returned on December 28, 2015. The National Oceanic and Atmospheric Administration (NOAA) antenna partners gradually were able to close the RF link on the space weather broadcast in between DSN or ESA tracks.

With the increased downlink rate, the 15 months of in-situ instrument space weather data on the SSR were finally able to be played back twice and verified that all recorded data was received.

On November $17^{\text {th }}$, the Ahead spacecraft bus reconfigurations were completed for returning to nominal science operations. After 454 days of side lobe and solar conjunction operations on Ahead, daily SSR playback, commanding, and tracking operations resumed. Daily science data return was gradually restored to prime science levels of 5 Gbits by December $28^{\text {th }}$.

Post conjunction, all instruments resumed nominal data collection with no ill effects from being powered down for several months. The HGA feed assembly temperature gradually returned to within tested operational limits, $<90^{\circ}$
C, with no ill effects from being overheated for over two years. Only one lingering issue resulted from the solar conjunction on the Ahead observatory. G\&C fine pointing losses and the magnitude of the attitude roll error increased slightly post solar conjunction. The SECCHI imaging instrument team reported that the magnitude of the attitude roll error was periodically affecting the Heliospheric images causing the post processing star-fitting algorithm to fail. To improve roll error performance, the $\mathrm{G} \& \mathrm{C} \mathrm{X}$-axis control bandwidth was adjusted. The G\&C Rate Merge capability, which enables rate measurements from all sensors to be combined to form a "better" rate estimate versus picking only one sensor as the source of the angular rate measurement, was also enabled, which reduced the noise on the $\mathrm{X}$-axis angular velocity estimate by $\sim 50 \%$. However, the cause for the changed G\&C behavior has not yet been determined. Wear on the reaction wheel bearings seems like a possible explanation.

\section{LOSS OF AND EFFORTS TO RECOVER THE STEREO BEHIND OBSERVATORY}

On October 1, 2014, after the Behind observatory had completed its on orbit testing prior to superior solar 
conjunction, communications were lost after the HCLTinitiated system reset was observed during a DSN 70 meter support with the radio science receivers recording. Data extracted from the DSN radio science receiver (RSR) data proved invaluable as it led to the understanding that the gyro failure caused the spacecraft to spin up and turn away from the Sun. Within a few hours, the battery drained, and the spacecraft became very cold freezing all propellant. A NASA Failure Review Board was convened and concluded that the loss of communications was due to a simultaneous double failure of G\&C sensors. Over time, the spacecraft could thaw and slow down allowing the solar arrays may get sufficient power. Therefore they recommended that the project continue to periodically try to reestablish communications with STEREO Behind [4].

On the $25^{\text {th }}$ attempt to re-establish communications with STEREO Behind, the DSN detected a strong downlink carrier at 2227 UTC on August 21, 2016 after contact was lost for over 22 months. At a spacecraft range of $2 \mathrm{AU}$, the observatory was found to be rotating slowly about its principal axis of inertia for which the uncontrolled attitude allowed some solar array input and continuous uplink and downlink communications on the LGA at emergency data rates. Over the next 22 continuous days, significant obstacles to recovery were overcome with a collaborative effort of the JHU/APL engineering team, NASA GSFC, DSN, Flight Dynamics Facility (FDF), GSFC Space Science Missions Operations (SSMO) scheduling, and Mission Operations teams. This consisted of:

- Reliably commanding a rotating spacecraft with uncontrolled attitude at a distance of $2 \mathrm{AU}$

- How to power on the spacecraft that was never designed to be off without collapsing the battery voltage

- Acquiring telemetry at $35 \mathrm{bps}$ from a spacecraft that is rotating with an uncontrolled attitude

- Warming a frozen propulsion subsystem with a degraded battery and limited solar array input with an uncontrolled attitude

- Configuring, loading, and verifying memory objects with very limited telemetry

- Conducting an autonomous momentum dump in the blind and transitioning to Control and Data Handling $(\mathrm{C} \& D H)$ standby mode and successfully receiving telemetry on the HGA indicating star tracker lock and decreasing system momentum.

However, system momentum level remained above the threshold for re-establishing attitude control with the reaction wheels. Due to the uncontrolled attitude, communication degraded and the last detection of the carrier was on September 23, 2016.
From the last telemetry received on September 18, 2016 and the telemetry assessment review held on February 24, 2017, main bus voltage is low, 3 out of 11 battery cells are bypassed, attitude remains uncontrolled, rotating about its principal axis of maximum moment of inertia. While likely all $\sim 42 \mathrm{~kg}$ of hydrazine remains and is frozen, both pressure transducers are not functioning. Earth Acquisition (EA) mode is enabled and autonomy is disabled. The battery charge rate is $\mathrm{C} / 10$. $\mathrm{RF}$ is configured for the $+\mathrm{Z}$ LGA at emergency data rates and the range of the expected best lock frequency (BLF) is known. Necessary command sequences have been tested to allow the peak power tracker in C\&DH standby mode to protect the battery. These command sequences will be loaded to EEPROM when the communications supports longer commands.

From the very limited telemetry (245 packets) received during the recovery attempt and many engineering team meetings, significant improvements were developed and tested on the hardware simulator which included:

- Data rates were optimized, based on RF link performance, by adding 4 new uplink rates and 16 new downlink rates.

- Use the -Z LGA as it provides $2 \mathrm{~dB}$ better performance than + Z LGA.

- A ground software process was developed to determine attitude and estimate solar array input using only Doppler data and ephemeris.

- Recover the Behind observatory in the higher C\&DH standby mode which allows use of the peak power tracking algorithm to better protect the degraded battery while warming propulsion subsystem.

> Should battery voltage subsequently collapse, upon power restoration, the spacecraft will autonomous promote back to $\mathrm{C} \& \mathrm{DH}$ standby mode.

- Minimize fault protection rule usage to only those that will assist with recovery operations.

- Minimize battery loading during and after the autonomous momentum dump used to re-establish attitude control.

- As a propulsion leak may have occurred, close propulsion latch valves after each thrusting operation to minimize propellant loss and undesired specific impulse from leakage.

- Refined the autonomous momentum dump options based extensive hardware testing and analysis

Use IMU1 for autonomous momentum dump as it will null rates about $\mathrm{Y}$ and $\mathrm{Z}$ axes better and results in rotating about $+\mathrm{X}$ axis pointed at Sun (power positive) 
$>$ Power reaction wheels on after all momentum dumps have completed.

$>$ Do not use IMU2 as it causes increased rotation.

$>$ Do not command a slow "rotisserie" roll rotation for sweeping LGA by the Earth for communications but rather cycle LGAs each hour.

Based on G\&C geometric analysis and GSFC modeling, daily recovery efforts began on August 21, 2017, and will continue through November 5, 2017. With significant support of the DSN, three different acquisition sequences are being utilized weekly to re-establish communications with STEREO Behind using a DSN 70 meter track:

1. $4 \mathrm{kHz}$ Sweep - consists of repeatedly sweeping a $4 \mathrm{kHz}$ uplink frequency range for which the BLF was found during the first recovery attempt. Commands are sent to power on the transmitter for 30 minutes. If no carrier signal is detected, the transmitter is powered off and battery recovery commands are sent consisting power off the avionics switched power and 1553 interface bus. This acquisition sequence is used 3 times each week.

2. Frequency Segmented $1 \mathrm{kHz}$ - To ensure command reception, the original faster frequency segmented acquisition sequence is used 2 days each week. This consists of an $18 \mathrm{kHz}$ frequency range divided into 18 , $1 \mathrm{kHz}$ segments, each swept at a $1 \mathrm{kHz}$ rate, stepping down in frequency and them back up again. During each segment, the MOC sends battery recovery commands on the first day and on the second day, carrier recovery commands. This was the acquisition sequence that originally detected the Behind downlink in August 2016.

3. $4 \mathrm{kHz}$ Sweep with Search Pattern - The DSN created a diamond shaped search pattern with 7 steps of 0.037 deg, dwelling 10.8 minutes/step. There are two diamond patterns of 4 steps to cover the area of the estimated ephemeris error. The starting point is offset $0.02 \mathrm{deg}$ for $100 \%$ uplink optimization. Repeating each diamond pattern accounts for the 30 minute round trip light time (RTLT). This search pattern acquisition sequence is used twice weekly during 3 hour supports, sending battery recovery commands at each step during the first day and on the second day, sending carrier recovery commands at each step.

\section{LESSONS LEARNED}

The significance of this work for future deep space missions, for which continuous fine pointing and/or a long superior solar conjunction is required, is that the mission must be designed to support it. The following lessons were learned for incorporation into future NASA missions.
1. Add value based requirements for the extended mission phase for NASA missions. NASA missions are designed for the duration of the prime science success criteria. They do not reflect the reality for average NASA observatory lifetime, which is typically greater than 9 years. During development, value based requirements should be discussed for continuing the mission beyond the prime science phase to include all subsequent mission significant orbital events. For example for STEREO, adding a single requirement for safe survival of the months long superior solar conjunction would have uncovered the following:

- HGA feed thermal issue

- Unmodifiable 3 day HCLT initiated system resets

- Star tracker power off for each system reset

- No RF margin on LGAs at a 2 AU range

2. Use a value based science data return requirement. The STEREO mission required an annual average of 5 Gbits/day per observatory as opposed to requiring a data return percentage. This simplified flight and ground software and DSN track time usage. Ground segment teams (DSN, MOT, and schedulers) always try to maximize data return. This allowed for a manageable, unattended track operations implementation with reduced MOT staff. It also provided project flexibility in responding to DSN schedule changes.

3. Use a decoupled instrument operations concept if possible. Instrument teams are responsible for their own instrument commanding and instrument health assessment for decoupled instrument operations. It requires prelaunch allocation of shared resources, i.e., power, thermal, pointing, bus bandwidth, SSR storage, etc. It significantly reduces MOC complexity and minimizes MOT phase E staffing.

4. Success of launch, phasing orbits, prime science and extended mission phases is due in large part to extensive prelaunch systems testing on each observatory.

5. Add a common emergency frequency for all NASA missions which would allow rudimentary emergency contact between any two NASA missions. Had this been in place, the NASA Mars Atmosphere and Volatile EvolutioN (MAVEN) observatory might have been able to send the one command per day required to keep the HCLT on either spacecraft from expiring. NASA is currently studying this possibility.

6. Counting on RLGs for very long missions is risky; include option to operate on an intermittent basis.

7. If a spacecraft has redundant IMUs, allow for cross strapping between individual units so as to permit full three-axis coverage using any combination of sensors capable of providing measurements along any three 
non-collinear axes. Further, individual IMU units should be oriented on the spacecraft in a way that permits maximum flexibility in combining outputs while covering three mutually perpendicular axes.

8. Operate the Star Tracker as cold as possible to accelerate reacquisitions.

9. Do not make the hardware command-loss timer (HCLT) unmodifiable. Rather, design the HCLT duration as an uploadable parameter. If this feature had been available, we would have set the timeout value to a large number to prevent the 35 system resets during the Ahead observatory blackout period. This action would have prevented the reaction \#3 wheel over-speed latch-up.

10. Allow the system reset hardware response to be modified post-launch, i.e., EEPROM, not fieldprogrammable gate array (FGPA). For example, STEREO's unmodifiable reset sequence turns the star tracker off. The powering on and subsequent slow star re-acquisition of the star tracker was a contributing cause of the Behind loss of communication anomaly.

11. Set the battery charge current to maximum at start-up power initialization and after going into low state of charge operation to allow maximum charging capabilities with available solar array power. This design change would improve the odds of recovering a spacecraft from a depleted battery condition.

12. Provide MOT the ability to easily and completely disable autonomy, using, say a short hardware command providing ability to cut-off autonomy at extremely low uplink bit-rates. Our inability to cut-off autonomy painfully bit us twice during the Behind recovery efforts, i.e., wheel spin-up, then inability to communicate.

\section{STEREO SCIENCE}

STEREO mission data have been utilized for over 1,300 studies in the refereed literature. It would not be possible to do justice to all the varied research that the mission has enabled. Here we provide just an overview of some the important science produced by the mission.

The scientific goals of the STEREO mission are centered around solar activity. In particular, the mission is focused on coronal mass ejections (CMEs), large magnetic disturbances that erupt off the sun and into the solar system, and solar energetic particles (SEPs), atomic particles that are accelerated to relativistic speeds by solar activity. Both of these phenomena form part of what is referred to as space weather, which can have significant effects on human technologies, including spacecraft, communications, and power systems. STEREO also studies the solar wind, the Sun's magnetized atmosphere that expands outward from the sun and into the solar system. The solar wind varies, exhibiting relatively low speed flows from regions in the Sun's atmosphere with a closed magnetic topology and higher speed flow emanating from coronal holes, regions in which the Sun's magnetic field opens out into the solar system. These high-speed streams can, like CMEs, create disturbances in Earth's magnetic field. Furthermore, the solar wind affects the propagation of CMEs, altering both their speed and trajectory.

\section{Coronal Mass Ejections.}

Coronal mass ejections are giant magnetized clouds of plasma that erupt off the sun at hundreds of kilometers per second, expanding to solar-system wide scales They are difficult to observe from the ground and were first discovered using space based coronagraphs, telescopes with occulting disks that cover the bright sun, making its much fainter outer atmosphere, the corona, visible. CMEs are associated with other eruptive phenomena, including solar flares, intense brightenings that occur low in the solar atmosphere. These are all due to releases of energy from the sun's complex magnetic fields. Like flares and sunspots the number of CMEs changes over the course of the sun's approximately eleven-year activity cycle, with about one every other day at solar minimum and 2-3 a day solar maximum [5]. CMEs usually take 1-4 days after eruption to arrive at Earth orbit, although occasional highly energetic ones can arrive in less than 24 hours. If oriented correctly the magnetic field of the CME can couple with Earth's magnetic field resulting in geomagnetic activity, including major aurora displays around Earth's magnetic poles. Geomagnetic disturbances can also result in problems with communications, navigation, spacecraft, and power systems. Thus, understanding CMEs, including their structure and how they propagate through the solar system is highly important in being able to better predict space weather.

STEREO's multiple points of view have greatly enhanced our understanding of CME structure and trajectories. In one of the first major results of the mission, STEREO data allowed us to confirm that CMEs conform to the general shape of a magnetic flux rope - consisting of helical field lines bending back towards the sun at both ends (see Figure 7). Before STEREO it was not clear if there were CMEs with different types of topology or if we were seeing the same basic structure from different points of view.

STEREO's suit of 5 imaging telescopes (Figure 8) has allowed us to image CMEs and other structures in the solar wind all the way from the sun to Earth orbit and combine these images with in-situ and radio data from the STEREO and other spacecraft. This has allowed us to bring together information concerning large-scale structures detected by remote sensing with local characteristics observed with in situ instrumentation. 


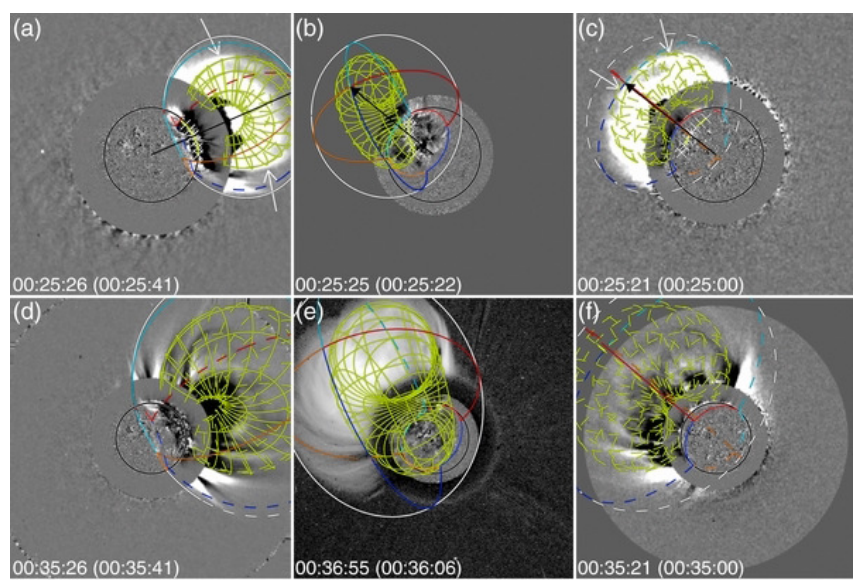

Figure 7. 3D reconstruction of CME with a flux rope model [6] superimposed on coronagraph images from two different times on March 7, 2012. from STEREO-A (left), SOHO (center) and STEREO-B (right). The model flux rope is shown in green and the associated shock wave is in blue. [7]. STEREO's additional points of view have made it possible to probe the three-dimensional structure of these solar storms. CAAS. Reproduced with permission.

STEREO has demonstrated the utility of a view to the side of the Earth-sun line to improve predictions of CME arrival times, an important goal in space weather forecasting. Multiple points of view are highly useful in determining the speed and trajectory of the CME. Even so simple a thing as determining if a CME is headed towards or away from the Earth can be difficult with only one point of view. There has been extensive research into methods for CME forecasting using STEREO data and studies have shown that predictions incorporating STEREO data can lead to improvements of up to half a day as compared to predictions based on a single view-point on the Earth-sun line [8]. This has led to efforts to provide space weather monitoring missions to the Earthsun L5 and/or L4 points.

Multiple points of view have allowed researchers to carefully map the three-dimensional trajectories of CMEs as they move through the solar system. This has allowed us to understand CME-solar wind interactions, for instance that the solar wind acts as a drag on CMEs, causing them to speed up or slow down to match the solar wind speed. Also, CMEs can be redirected by structures in the solar wind such as coronal holes, causing CMEs that are initially directed out of the ecliptic to trend back down to where they are more likely to impact Earth.

\section{Solar Energetic Particles}

Solar energetic particles are particles (electrons, protons, and heavier ions) that move at relativistic speeds. These particles present a danger to space hardware. Generally, SEPs with narrower latitudinal distributions (generally less than $30 \mathrm{deg}$ ) and shorter more impulsive time profiles (a few hours) are thought to have been accelerated by solar flares in the sun's atmosphere, whereas ones with greater spatial distribution and more gradual, multi-day time profiles are thought to have been accelerated in shock fronts ahead of CMEs [9].

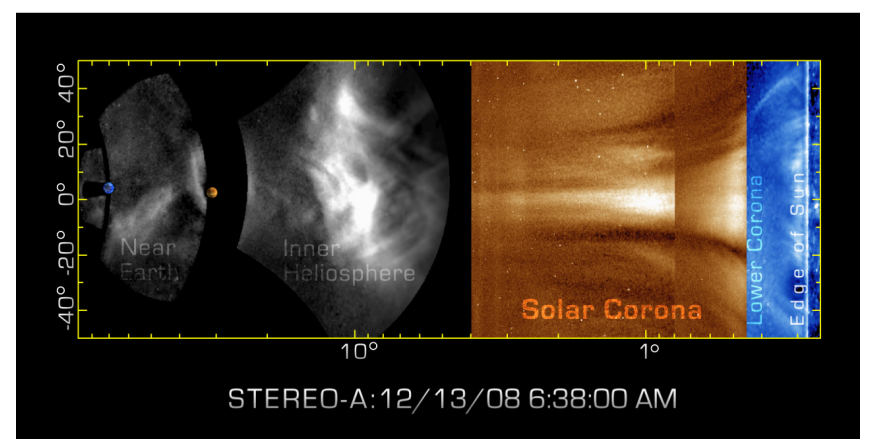

Figure 8. Each STEREO spacecraft contains five telescopes that make it possible to track CMEs all the way from the sun to Earth orbit. These images from STEREO-A show a CME moving out from the sun (in blue on the right) passed Venus and Earth (represented by the brown and blue dots respectively on the left) Credit: NASA/Goddard Space Flight Center/SwRI/STEREO.

It is thought that such high velocity charged particles should move outward from the sun along magnetic field lines shaped into a spiral (known as the Parker spiral) by solar rotation and the expansion of the solar wind (see Figure 9). It should be possible to determine the acceleration sites of SEPs by tracing along the Parker Spiral from the observation site. However, STEREO's multi-point measurements have confirmed early observation from the Helios mission that SEP's are often spread over much greater latitudes than expected from particle transport along the Parker sprial. Figure 9 shows a case in which spacecraft located at a wide range of longitudes registered SEPs within a narrow time range that is difficult to explain by simple particle diffusion. This is one of many such examples.

STEREO's in-situ instrumentation has allowed us to study the longitudinal distribution, composition, energies, and anisotropies of SEPs, along with their source regions, resulting in a more complete understanding of how SEPs are accelerated and transported through the solar system.

STEREO's radio instrumentation has also been important for studying these particles. Type II radio bursts are formed at the shock fronts that accelerate gradual SEPs, while type III bursts are produced by relativistic electrons streaming along magnetic field lines in the solar wind, see Figure 10.

\section{STEREO 360}

STEREO has led to a more complete picture of space weather by providing us information concerning solar activity beyond just the Earth-sun line. Prior to STEREO our main sources of space weather information were relatively close to Earth, either in Earth orbit or at the Earth- 
sun L1 point. As the STEREO spacecraft moved towards the far side of the Sun, STEREO was able to provide information concerning space weather through an entire 360 degrees around the Sun, see Figure 11. (a)

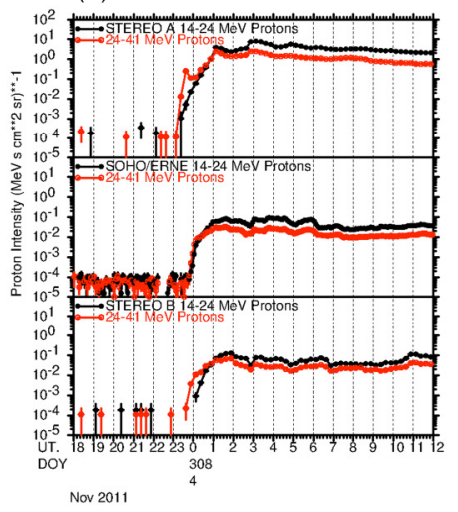

(b)

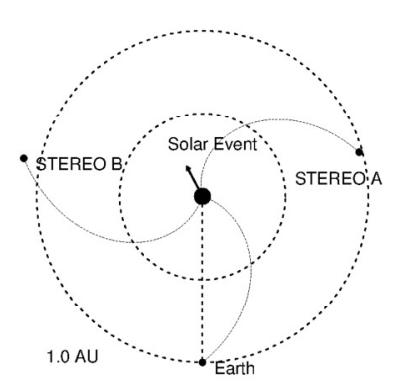

Figure 9. Solar energetic particle (SEP) event of 2011 November 3 observed across a broad range of longitudes [10]. The diagram shows the locations of STEREO-A and $B$ and SOHO (near Earth) and the spiral lines show the expected magnetic connections of the spacecraft to locations in the solar surface and in the solar wind. The arrow shows the direction of the CME. STEREO-A, the best connected of the spacecraft, showed the strongest SEP fluxes, but all three measured SEPs soon after the eruption. Figure courtesy I. Richardson [10].


Figure 10. Left: CME with preceding shock wave imaged by a coronagraph on STEREO-A. Right: Radio bursts observed by STEREO-A/WAVES, including Type II burst produced by the shock. The plot also shows Type III bursts that trace electron beams as they move along magnetic field lines and Type IV bursts emitted by trapped particles in loops in the corona. Figure courtesy N. Gopalswamy, NASA/GSFC.

Starting in 2011 images from STEREO combined with those from SDO in Earth orbit allowed us to image the entire solar disk. The sun takes approximately 27 days to rotate on its axis. During this time active regions on the sun, the source of the most intense solar activity, can evolve significantly. Imaging and in-situ measurements on the far side of the Sun can thus provide advanced warning of new or intensified active regions or coronal holes before they rotate onto the Earth facing side of the Sun and affect space weather near Earth.
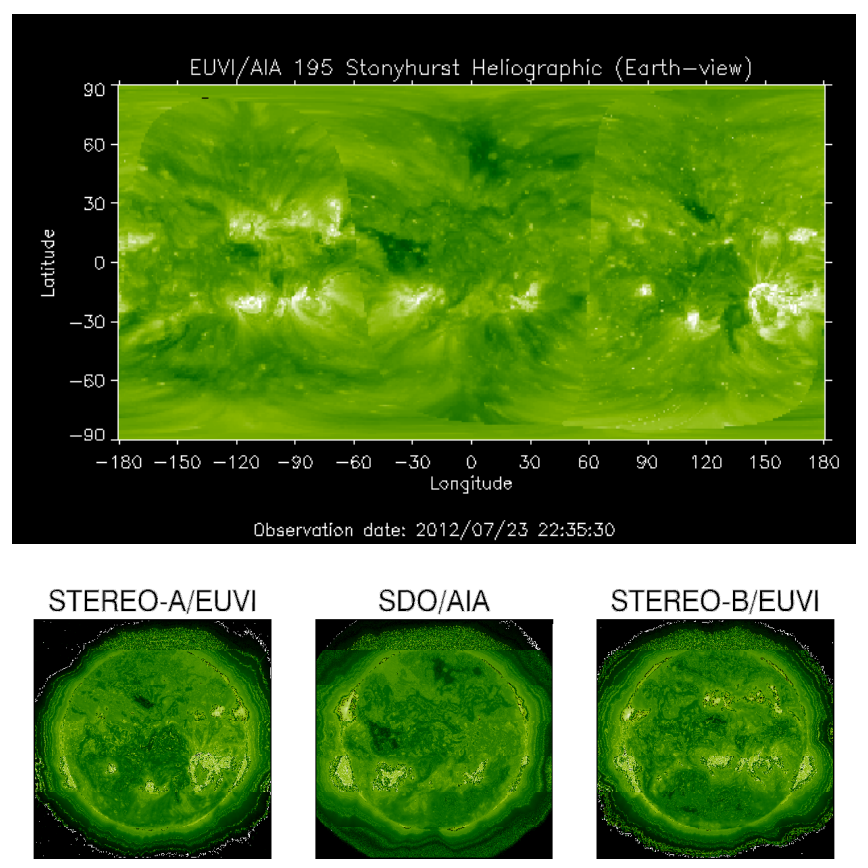

Figure 11. Top: The entire solar corona at $195 \AA$ as observed from three points of view by STEREO-A, Solar Dynamics Observatory (SDO), and STEREO-B on July 23, 2012, shown in the bottom row. At the time, the three spacecraft were approximately 120 degrees apart.

STEREO's presence on the far side of the sun provided us with a much more complete understanding of solar activity from all parts of the sun. This is important both for protecting NASA assets throughout the solar system and also for obtaining a more complete picture of the totality of space weather events. For instance, in July of 2012 STEREO-A was buffeted by one of the most energetic CMEs of the space age (see Figure 12). Multi-point observations from STEREO-A and B and SoHO revealed that this CME was the culmination of a string of CMEs, and showed some of the highest velocities on record accompanied with intense SEPs [11]. Studies of the event indicate that an earlier $\mathrm{CME}$ preconditioned the solar wind so that the later $\mathrm{CME}$ experienced relatively limited deceleration between the sun and 1 AU [12].

STEREO provided the opportunity to analyze in depth this event, and gives us greater insight into the frequency of such extreme weather events, which could have substantial affects when directed at Earth [13].

Observations of the far side of the sun have also allowed us to research in more depth the origins of CMEs. For example, CMEs are usually associated with solar activity 
that can be seen by imaging the sun directly, including solar flares, coronal dimmings, and prominence eruptions. However, there have been reports of "stealth" CMEs that do not seem to be accompanied by signatures in the low corona. With only one point of view it can be difficult to determine if such a CME is headed towards or away from the observer, making it impossible to determine if the CME truly has no signature low in the corona, or is simply erupting on the far side of the sun. STEREO data have made feasible studies of this topic, which has ramifications for space weather prediction as well as for our general understanding of CME initiation. Recent research using STEREO data has shown that even CMEs previously reported as stealth CMEs do show some sort of signature in the low corona, although sometimes it is quite faint. The CMEs which show minimal signatures tend to be relatively slow moving, and it has been suggested that they are initiated from much higher in the corona than usual, resulting in low acceleration [14].
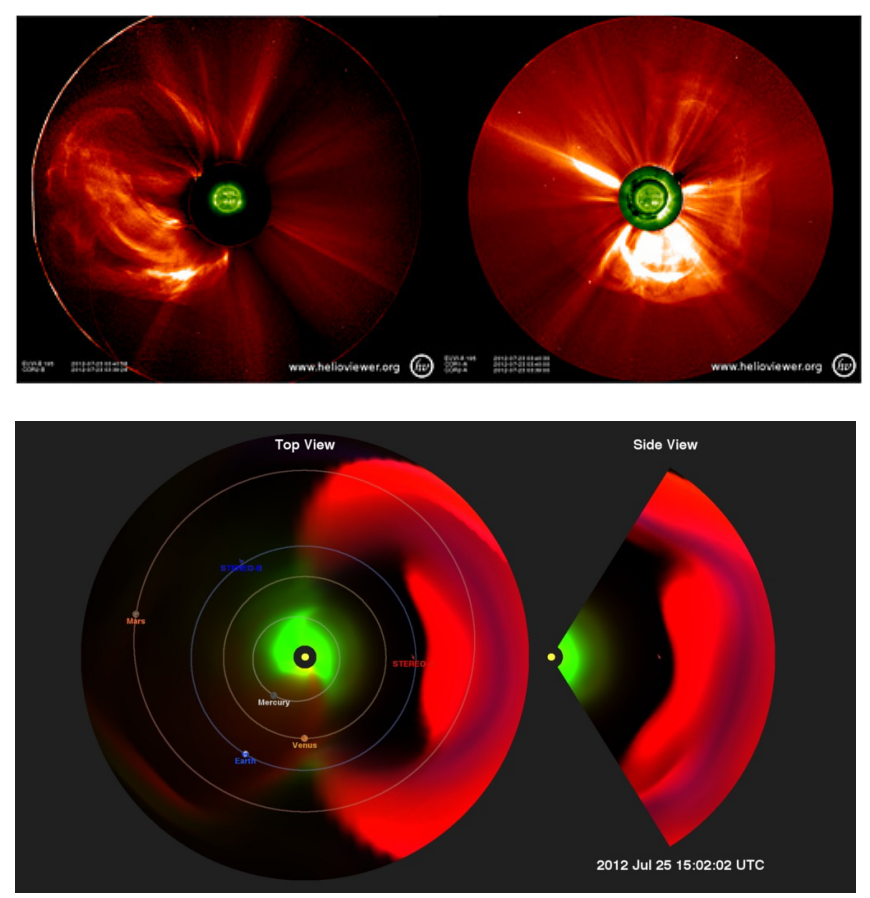

Figure 12. Top: Images of the CME erupting on the July 23, 2012 as observed STEREO-A (left) and STEREO-B (right) [13]. This CME, one of the fastest on record, was directed away from Earth and we would have little understanding of its magnitude without data from STEREO-A, which was directly in its path. Bottom: Simulation of the CME based on STEREO and SOHO data. To the left is a view of the ecliptic plane seen from the north. To the right is the view in the ecliptic of the CME passing STEREO-A. The green shows the density variations and red the temperature variation associated with the CME. Credit: NASA GSFC SVS.

\section{Conclusion}

With STEREO Ahead and Behind, we have the first stereoscopic view of complex and dynamic solar wind structures and larger coronal mass ejections, the first views of the backside of the Sun, and vastly improved our multipoint detection of energetic particles within the heliosphere. The unique 360 degree perspective offered by the two STEREO spacecraft has transformed our understanding of how the Sun operates both in terms of the underlying physical processes but also in terms of dramatically improving our capability in space weather prediction. Not only has the STEREO mission advanced our understanding of solar and heliospheric physics, but the unique challenges of the mission itself have considerably advanced our understanding of the technical hurdles in operating missions well beyond the primary science phase.

\section{ACKNOWLEDGEMENTS}

Many thanks to the science, engineering, navigation, scheduling, DSN, ESA, mission operations, and management staff for all their dedicated support to make the first 10 years of STEREO mission so successful.

\section{REFERENCES}

[1] M.L. Kaiser, T.A. Kucera, J.M. Davila, O.C. St. Cyr, M. Guhathakurta, E, Christian Space Science Reviews 136, p. 5-16, 2008, doi: 10.1007/s11214-007-9277-0

[2] D.A. Ossing, D.W. Dunham, J.J. Guzman, G.A. Heyler, J.E. Eichstedt, and H.D. Friesen, "STEREO First Orbit and Early Operations", Astrodynamics Specialist Conference, Mackinac Island, MI, August 19-23, 2007. Paper AAS 07377.

[3] D.A. Ossing et al, "STEREO Superior Solar Conjunction Mission Phase, 2017 IEEE Aerospace Conference, Big Sky, MT, March 4-11, 2017, Paper 2461

[4] R.R. Harman et al, "Solar Terrestrial Relations Observatory BEHIND (STEREO-B) Loss of Communications Failure Review Board Report", NASA GSFC, March 20, 2015.

[5] A. Vourlidas , L.A. Balmaceda, G. Stenborg, \& A. Dal Lago, ApJ 838 141, 2017 doi: 10.3847/1538-4357/aa67f0

[6] A. Thernisien, A. Vourlidas, and R. Howard, ApJ, 652, p. 763, 2006, doi: 10.1086/508254

[7] R.-Y. Kwon, J. Zhang, and O. Olmedo, ApJ, 794, 148, 2014, doi: 10.1088/0004-637X/794/2/148

[8] R. Colaninno, A. Vourlidas, and C.C. Wu, JGR (Space Phyiscs), 118, 6866, 2013, doi: 10.1002/2013JA019205 
[9] M. Desai, and J. Giacalone, Living Rev. Sol. Phys. 201613 p. 3. 2016, doi:10.1007/s41116-016-0002-5

[10] I.G. Richardson, T.T. von Rosenvinge, H.V. Cane et al. Sol Phys 289: 3059, 2014, doi: 10.1007/s11207-014-0524-8

[11] C.T. Russell et al, Astrophysical Journal, 770, 38 2013, doi:10.1088/0004-637X/770/1/38

[12] Y.D. Liu et al. Nature Communications 5 3481, 2014 doi:10.1038/ncomms4481.

[13] D.N. Baker et al. 2013 A Major Solar Eruptive Event in July 2012: Defining Extreme Space Weather Scenarios, Space Weather, S11, 585, 2013, doi:10.1002/swe.20097

[14] N. Alzate and H. Morgan, 2017 ApJ 840 103, doi: 10.3847/1538-4357/aa6caa

\section{Biography}

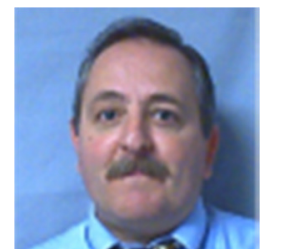

Daniel A. Ossing received a B.S. in Marine Engineering, from the Massachusetts Maritime Academy in 1982 and an M.S. in Electrical Engineering from The Johns Hopkins University in 1989. As a member of the Principal Professional Staff he has been with the JHU/Applied Physics Laboratory for more than 32 years. He has been the mission operations manager of NASA's STEREO mission since 2008. Concurrently, he was also the mission operations manager for the first JHU/APL dual cubesat mission, MBD. He has conducted mission operations for the ISRO Chandrayaan-1, NASA LCROSS, NASA TIMED, and MDA MSX missions. His career started as an Engineering Officer for ARCO Marine transporting Alaskan north slope crude oil.

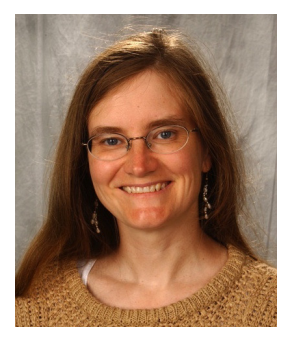

Therese Kucera received a B.A. in Physics from Carleton College in 1987 and a PhD in Astrophysical, Planetary and Atmospheric Sciences from the University of Colorado, Boulder in 1993. Since that time, she has worked at Goddard Space Flight Center, and has served on the teams of the SOHO, SPARTAN 201, and STEREO missions. She is currently STEREO Project Scientist.

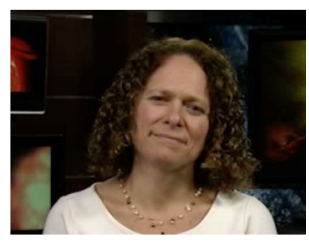

Georgia. de Nolfo received her B.S. and M.S. from the University of Chicago and her PhD in 1997 from Washington University, St. Louis. She spent 4 years as a postdoctoral scholar at the California Institute of Technology and as a National Research Council Fellow at NASA/Goddard. Since then Dr. de Nolfo has worked in experimental astrophysics at NASA/Goddard focusing on instrument development, mission support, and data analysis of energetic particles. Dr. de Nolfo has served on numerous astrophysics teams (e.g., high-altitude balloon-borne missions, CALET) in addition to the Advanced Composition Explorer (ACE) team. Currently Dr. de Nolfo is providing instrument hardware for several upcoming CubeSat missions including a terrestrial gamma-ray flash payload, TRYAD, and an astrophysical gamma-ray burst payload, BurstCube. Dr. de Nolfo is Deputy Project Scientist for the ACE and STEREO missions.

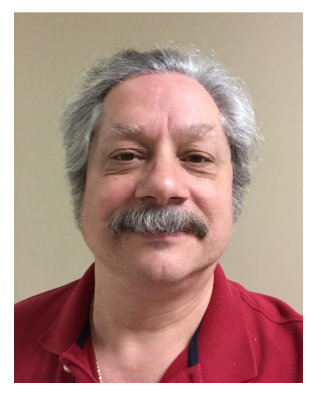

David A. Quinn received a B.S. in Aerospace Engineering from the University of Maryland at College park in 1983 and a M.S. in Mechanical Engineering from George Washington University in 1998. He has been with NASA/GSFC for more than 28 years. His career at GSFC started with leading the Pointing Control System group for the Hubble Space Telescope during the launch, deployment, operation and first servicing mission. He was involved in operations, mission design, analysis, and flight dynamics for the Landsat-4, 5, \& 7, TERRA, LRO, LCROSS, LADEE, and TESS missions. During his time at GSFC, he co-invented the formation flying technique fundamental to multispacecraft control efforts at GSFC. He also conceived and developed the CEGANS (Compound Eye GPS Attitude and Navigation Sensor) concept for which he received a U.S. patent in 2003. He authored a proposal for the CrossEnterprise Technology Development Program (CETDP) for tethered formation flying development and was awarded funding to serve as the Principle Investigator for tethered formation flying development in support of the SPECS mission concept. Currently, he is the Mission Director for the NASA/GSFC Space Science Mission Operations branch where he has primary operations responsibility for the NASA TIMED, STEREO, Van Allen Probes, and SOHO missions. 